\title{
3DIR: three-dimensional information retrieval from $3 D$ building information modelling environments
}

\author{
P. Demian ${ }^{1}$, K. Ruikar ${ }^{1} \&$ A. Morris ${ }^{2}$ \\ ${ }^{1}$ School of Civil and Building Engineering, \\ Loughborough University, UK \\ ${ }^{2}$ Centre for Information Management, \\ Loughborough University, UK
}

\begin{abstract}
More and more information is being packed into Building Information Models (BIMs), with the 3D geometrical model serving as the central index to lead users to the many other types of building information. The Three-Dimensional Information Retrieval (3DIR) project investigates information retrieval from such environments, where information or documents are linked to a 3D artefact. In these situations, the 3D visualisation or 3D geometry of the building can be exploited when formulating information retrieval queries, computing the relevance of information items to the query, or visualising search results. Following reviews of literature in $\mathrm{BIM} / \mathrm{CAD}$ and information retrieval, a clear gap was identified in the practice of information retrieval from BIM/CAD systems. End users were consulted to ascertain the precise user requirements in such an information retrieval system. Scenario-based design was adopted to design a software prototype. The 3DIR system was developed as an add-in under the Autodesk Revit BIM platform. The 3DIR prototype creates an index of all text data attached to the 3D model. The user is able to search for information by selecting specific 3D objects, by keyword and by specifying particular 3D regions of the model. Relationships between 3D objects are also used to rank search results. Search results are displayed by highlighting 3D objects in the 3D model. Findings from the evaluation of the prototype demonstrate its usefulness but suggest directions for future development. It is concluded that a tight coupling between text-based retrieval and the 3D model is extremely effective in 3D BIM environments. Keywords: information retrieval, document management, $3 D$ models.
\end{abstract}




\section{Introduction}

In building modelling environments, more and more information is being crammed into $2 \mathrm{D} / 3 \mathrm{D}$ building and product models. This is particularly true given the rise of Building Information Modelling (BIM) (Eastman et al. [1]). The ThreeDimensional Information Retrieval (3DIR) project investigates information retrieval from these environments, where information or documents are linked to a 3D building model. In these situations, the 3D visualisation or 3D geometry of the building can be exploited when formulating information retrieval queries, computing the relevance of information items to the query, or visualising search results. Managing such building information repositories in this way would take advantage of human strengths in vision, spatial cognition and visual memory (Lansdale and Edmonds [2], Robertson et al. [3]).

Information retrieval is associated with documents, although a critic might argue that documents are relics from the pre-BIM age that are no longer relevant in the era of BIM. However, the challenge of information retrieval is pertinent whether we are dealing with documents which are coarse grains of information or building object parameters/attributes as finer grains of information. As noted in section 2.2, Demian and Fruchter [4] demonstrated that traditional retrieval computations can be applied with good results to 3D building models where textual or symbolic data are treated as very short documents.

This paper describes the findings of the 3DIR project whose aim was to develop an information retrieval toolset for documents/information linked to 3D building models. This aim was achieved through the following objectives:

- To review the applicable techniques from the fields of BIM, information retrieval and information visualisation, particularly those techniques which exploit strengths in visual human cognition.

- To document formally the requirements in such a software toolset.

- To develop a software prototype and evaluate it, thereby validating the proposed theoretical coupling between 3D geometry and linked nongeometric information.

\section{Background and related work}

Building design, construction and operation are information intensive activities. For example, even over a decade ago in the UK construction industry, on average, one computer-aided design (CAD) document was produced for every $9 \mathrm{~m}^{2}$ of building floor space (Gray and Hughes [5]). Several others (Leslie [6], Veeramani and Russell [7], Ugwu [8]) have reported on the problem of "information overload" in the construction sector.

Information retrieval techniques have been used in civil engineering to retrieve reusable designs [4] and to retrieve contextual information from past projects to improve the accuracy of future cost estimates [9]. Beyond text, Brilakis and Soibelman [10] automatically identify particular features in construction site photographs with a view subsequently to using information retrieval techniques to manage photograph collections. Bridging textual and geometric content, Caldas et 
al. [11] propose techniques for automatically classifying construction documents based on project CAD components. Lin and Soibelman [12] augment standard information retrieval techniques with formal representations of domain knowledge to improve the performance of a search engine for online product information. Rezgui [13] similarly uses domain knowledge to formulate an ontology that informs the indexing and retrieval of construction content.

None of the studies encountered in the literature specifically exploit 3D data and $3 \mathrm{D}$ visualisations for information retrieval. This approach lies at the intersection of four academic fields: (1) BIM and CAD, (2) information retrieval, (3) information visualisation and (4) human cognition and how particular strengths can be exploited.

\subsection{BIM and CAD}

The state of the art in digital content management in building design and construction projects is being transformed by the emergence of Building Information Modelling [1]. Whereas CAD models classically attempted to model the geometry of buildings or building components in two or three dimensions (e.g. Eastman [14], Emmitt and Ruikar [15]), Building Information Models attempt to model nongeometric content as well. This content includes the nongeometric attributes of physical building components (such as the cost of a component) as well as nongeometric entities. For example, Building Information Models can include entities to model the processes of design (Austin et al. [16]) and construction (Koo and Fischer [17]) and the organizations (i.e. teams and individuals) that execute those processes (Kunz et al. [18]). In addition, building information modelling is not limited to the design and construction phases but can be extended to cover the entire life cycle of constructed facilities, from briefing/programming, through design, to facilities management and even disposal.

In the context of the 3DIR project, it is noteworthy that, although as noted above CAD and BIM models nowadays include both geometric and nongeometric information, the geometric 3D model of the building is central, and is expected to serve as a visual index that leads to the additional nongeometric content. This approach often fails because such systems do not exploit human abilities in spatial cognition and visual memory. Nongeometric content does not leave enough information scent [19] in the geometric CAD model that enables the information forager to find it. This concept served as an important point of departure for the 3DIR project.

\subsection{Information retrieval}

Information retrieval (IR) is concerned with systems that help users to fulfil their information needs, in particular computations to quantify the relevance of information items based on user queries [20]. Demian and Fruchter [4] demonstrated that traditional IR techniques could be applied to retrieve information from Building Information Models and product models; the semantic information attached to $3 \mathrm{D}$ objects could be treated as very short 
documents and standard text document computations employed, giving reasonable retrieval results. As noted in the introduction to section 2 and in section 2.1, information retrieval has recently been applied in managing the vast volume of information accumulated in building design, construction and operation.

\subsection{Information visualisation}

Visualisation has been defined as "the use of visual representations to amplify cognition" (Section 1 of [21]). Information visualisation (IV) in particular refers to the visualization of abstract data, unrelated to physical space. Such data (e.g. financial data, abstract conceptions, hierarchical and network data structures) have no obvious spatial mapping. One important branch of IV is the visualisation of collections of documents (Section 6 of [21]). Efforts to visualise document collections range in scale from visualising the whole internet (Section 6.1 of [21]), through visualising smaller document collections in information workspaces (Section 6.2 of [21]), to visualising an individual document (Section 6.3 of [21]). In construction, Wu and Hsieh [22] identified the lack of a single interface which combined and visualised the information from the disparate project sources as an important cause of work breakdowns. They go on to propose the Project Information Integration Management Framework (PIIM Framework), operationalised in a software prototype in which data is presented in conjunction with the 3D model. Shaaban et al. [23] propose different approaches for the application of IV in architecture. They present the task-driven approach as the most effective, which places the user's task and information needs at the centre, and considers his/her visual and cognitive processing.

\subsection{Human cognition}

Applying IV and IR in BIM environments requires an awareness of human visual cognition. Managing such document or information repositories linked to a 3D artefact can exploit human strengths in vision, spatial cognition and visual memory. In studying the psychology of personal information management, Lansdale [24] observed the importance of visual properties in the acts of recall, recognition and categorisation. With this understanding of the psychological fundamentals of information management, Lansdale and Edmonds [2] went on to develop a computer system for filing information based on a personal history of events; importantly, this system allowed for search and recognition based on visual attributes. In trying to establish a stronger link between psychological experimentation and system development, Lansdale et al. [25] developed theories of spatial cognition and applied them to the design of pictorial databases which allow the user to search by spatial or visual queries. Similarly, early research by Robertson et al. [3] showed that users were able to retrieve electronic documents much more effectively when thumbnails were arranged visually than when documents attributes were listed textually. More recently, Christmann et al. [26] performed user tests with prototype interfaces for browsing photographs and found that interfaces were more effective when they used a 3D layout with perspective than a simple 2D visual layout. 
The literature reviewed in section 2 highlights the promise of systematically applying IR and IV techniques in BIM environments to exploit human cognitive strengths and facilitate more effective information management.

\section{Research method}

Following reviews of literature as presented in section 2, a clear gap was identified in the practice of retrieving information from $\mathrm{BIM} / \mathrm{CAD}$ systems. To ascertain the precise user requirements of such an information retrieval system, workshops were convened at a large multinational contractor and a renowned architectural practice in London. Scenario-based design (Rosson and Carroll [27]) was adopted to design a software prototype. Data collected and analysed from these workshops were fed into the scenario-based design. The 3DIR system was developed as an add-in under the Autodesk Revit BIM platform. This was later presented to the industry partners in evaluation workshops. Although the ultimate aspiration is for any software development to remain platform independent and avoid favouring any particular commercial BIM environment, it was found that the Autodesk Revit Application Programming Interface (API) provided excellent opportunities for development and research prototyping.

\section{Needs analysis}

The ICT Director of "Architects", an international architecture practice based on London, was interviewed. The ICT Director spoke strategically about the shift from CAD to BIM (and, indeed, from hand drafting to CAD), the cost of software, the potential productivity gains and measuring the Return on Investment of these new tools. "Architects" had just adopted a new commercial BIM platform and, despite the software being perceived as extremely expensive, the productivity gains were evident: "it's taken a fraction of the time to produce the information we would normally produce with 2D drawings".

Following the interview with the "Architects" ICT Director, two architects joined the conversation and a focus group discussion was held. The architects echoed the productivity gains enabled by the new BIM platform. They noted that the BIM information architecture enabled much more information to be included almost effortlessly in the model. The information bearing capacity of the new 3D models was used as an important communication medium for collaboration. This contrasted with the pre-BIM days described by the ICT Director: "in those days, we could still put some data into our models, but nobody did it because we had nobody to share it with". Beyond including data within models, all focus group participants acknowledged the difficulty of linking external documents to BIM models. If those links were in place, however, the focus groups could clearly see the possibilities for improved information retrieval.

In addition to the interview and focus group at "Architects", a focus group was convened at "Contractor", a major UK contractor. The participants from "Contactor" did not feel that "documents" were going to remain relevant in the new era of BIM. They did acknowledge that models were becoming more and 
more information-rich, but not with traditional documents. Interoperability was an urgent issue which emerged repeatedly in that focus group. "Contractor" teams extensively used extranets on their projects. The participants identified the disconnection between 3D models and other project documents in extranets as a major obstacle to retrieving information, either from the documents or from the 3D models. Models in the extranet rarely contained links to other external information.

\section{Prototype development}

The needs analysis exposed the complexity of information management in BIM environments. The assumption made at the outset of the 3DIR project was that links existed in standard practice between 3D components and textual information or documents. The needs analysis demonstrated this this assumption was questionable, although the situation rapidly changed as the research progressed. Even though links between documents and 3D components remain rare, as noted from the literature: the textual, numerical or symbolic parameters given to $3 \mathrm{D}$ components in most BIM information architectures can be considered as non-3D information linked to $3 \mathrm{D}$ components. Isolating the challenge of exploiting such links for information retrieval, the following salient requirements (R) were distilled from the needs analysis:

R1. When formulating queries, users need the ability to search by keyword, 3D volume, by selecting a set of components from the model, or by any combination of these.

R2. When selecting a component or set of components from the model within which the user wishes to search, users would like the option of searching beyond this selection, based on relationships between components.

R3. When visualising search results, users need to retain the standard text-based listing, but would also like search results somehow superimposed on the 3D model.

These requirements emerged when the findings from the needs workshops were used to formulate fictional archetypal problem scenarios (Rosson and Carroll [27]). Those in turn were developed into activity scenarios, information scenarios and interaction scenarios, culminating in a usability specification. Through this process, requirements R1, R2 and R3 were translated into the following corresponding system usability specification items (S):

S1. Multiple search modes are needed:

a. Clicking on a single component or collection of components should display all the textual information (whether parameters or external documents) linked to that/those component(s).

b. The system should allow the user to filter the search by keyword, by selecting desired 3D components or by specifying a 3D volume.

S2. "Hops function": with a single component or collection of components selected, the system should give the user the option of searching or "hopping" outside this selection to related 3D components up to a specified maximum number of hops away. Search results can be ranked by "hops". 
S3. Text search results listing is needed, together with as many visual representations of search results as the API allows:

a. Text listing.

b. Retrieved 3D components are "selected".

c. Retrieved 3D components are isolated, i.e. all other components in the model are temporarily rendered invisible.

d. Retrieved 3D components are highlighted by insetting a phantom coloured shape above them. The colour of the shape can be used to denote the type of information retrieved (i.e. format of file or type of parameter) and the size of the shape can be used to denote the relevance according to the text retrieval computation.

A prototype was developed under the Autodesk Revit platform. Revit is a common commercial BIM platform and, as noted in section 3, upon reviewing common BIM platforms, was found to have a powerful Application Programming Interface. The source code was writing in C\# using the Revit .NET API. The Apache Lucene open source library was used for the text indexing and search functions.

\section{The 3DIR prototype}

3DIR is developed as an add-in to Revit, and therefore (once installed) appears in the add-ins ribbon of the standard Revit interface. The 3DIR toolbar consists of three tools: Index, Search and About. The first step when searching a building model using 3DIR for the first time is to "index" the model using that icon on the 3DIR toolbar. This will create an index of all text terms from the 3D object parameters or linked text documents. Once an index has been created, the "Search" tool can be used which brings up the dialogue box shown in Figure 1.

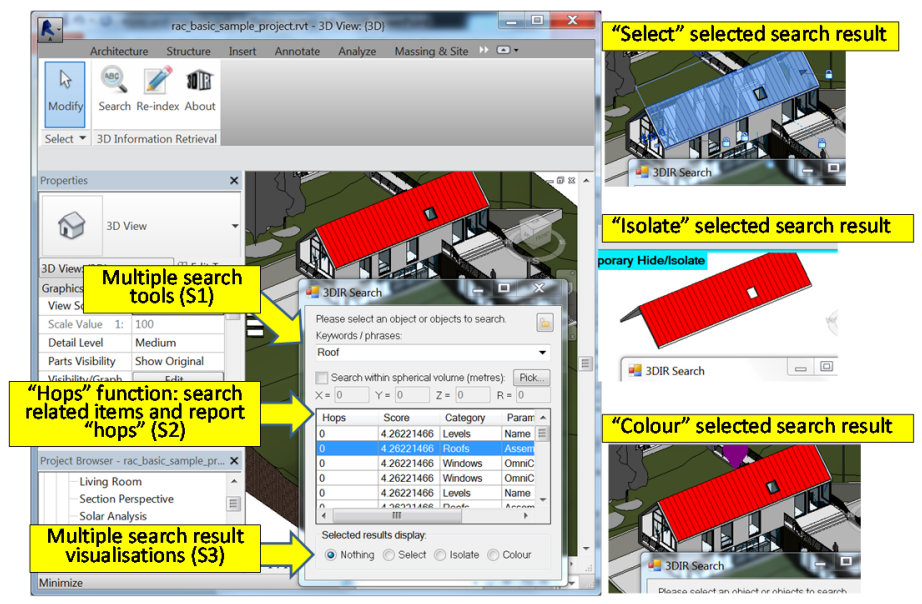

Figure 1: The 3DIR tool. Screenshots showing the interface and the software functions satisfying the specifications (S) which emerged from the scenario-based design. 
As the user enters keywords in the text box, search results are listed in real time in the dialogue box. In the example shown in Figure 1, the keyword "roof" is entered and search results listed related to the roof of the model. The search can be processed either on the whole building model if nothing is selected, or limited to the selected objects. As the object selection in the model changes, the search results are updated dynamically. The user is also able to limit the search to a spherical volume. Selecting a search hit from the list (e.g. the "Roof Assembly" item) will optionally "select" (or highlight) the Revit element containing that search term (i.e. the red roof graphic from the model), "isolate" it (i.e. temporarily hide all other items in the model) or identify the object by displaying a coloured balloon next to it in the building model. Figure 1 shows an example search for "roof". The three options for displaying the selected search result(s) appear on the right hand side of the figure.

\section{Prototype evaluation}

A further workshop and demonstration was held at the offices of "Architects" to evaluate the prototype and validate the principle of tighter coupling between the 3D model and nongeometric information. The feedback was positive, although several improvements and future developments were proposed. The three participants were asked to complete a simple software usability assessment by Brooke [28]; their scores are shown in Figure 2. It can be seen that the participants generally agreed with the positive statements and disagreed with the negative.

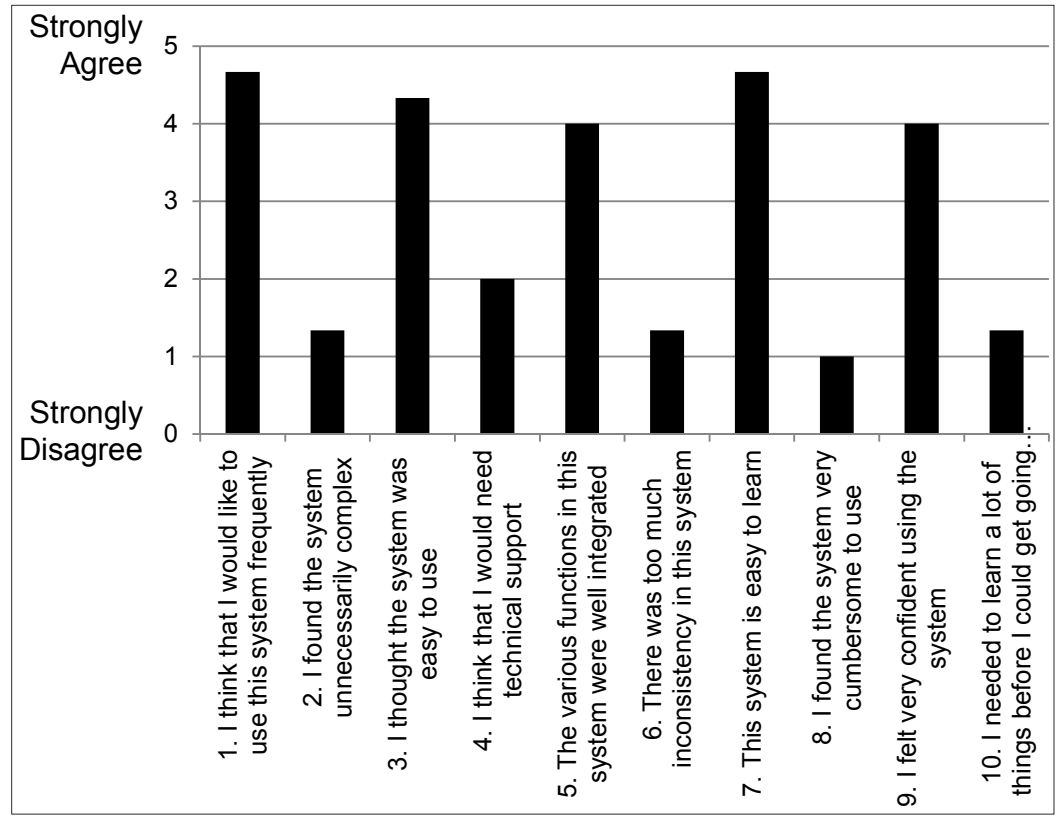

Figure 2: Interface usability scores (3 participants). 
It was felt by the "Architects" team that the system was "very useful at what it attempted to do". The information inside the model was often not textual, and links to external text documents remained rare. The future development most strongly proposed by the participants was to establish such links automatically, particularly links to email repositories to record design rationale as the model developed.

\section{Conclusions}

The 3DIR prototype creates an index of all text data attached to the $3 \mathrm{D}$ model (mostly as text properties of 3D objects). External text documents linked to 3D objects (using, for example, the "URL" type of object property in Revit) are also indexed (but those are rarely established in practice). The user is able to search for information by selecting specific 3D objects, specifying a spherical region of the model and/or entering search keywords. To compute relevance based on textual data, the Vector Space Model is used through the Apache Lucene library. Relationships between 3D objects are also used to filter and rank search results, whereby relevant information items linked to a related 3D object (rather than information linked directly to a 3D object selected by the user) are still retrieved but ranked lower. Search results are displayed by highlighting 3D objects in the 3D model. Findings from the evaluation of the prototype demonstrate its usefulness but suggest directions for future development. It is concluded that a tight coupling between text-based retrieval and the 3D model is extremely effective in $3 \mathrm{D}$ design environments.

\section{Acknowledgements}

Our colleague Dr Ann O'Brien is an original member of the 3DIR team and contributed to the work presented here but unfortunately passed away before this paper was written. The 3DIR project was supported by a Brian Mercer Feasibility Award from the Royal Society, with further support from the Enterprise Office at Loughborough University and funding from University's Higher Education Innovation Fund. Autodesk provided support through membership of the Autodesk Developer Network. Software development for the prototype was by Neil Sutton at Adris, part of the Graitec group. Loughborough University alumnus Tom Claridge wrote his undergraduate dissertation in the topic of 3DIR, and in the process provided research support for the project. The 3DIR team is grateful to the contractor and architecture offices for their support during the requirements gathering and prototype testing phases of the work. 3DIR is available for free from the Autodesk Exchange for Apps at: https://apps.exchange.autodesk.com/RVT/en/ Detail/Index?id=appstore.exchange.autodesk.com\%3a3dinformationretrievalforr evit_windows32and64\%3aen. 


\section{References}

[1] Eastman, C., Teicholz, P., Sacks, R., \& Liston, K. (2011). BIM handbook: A guide to building information modeling for owners, managers, designers, engineers and contractors. John Wiley \& Sons.

[2] Lansdale, M., \& Edmonds, E. (1992). Using memory for events in the design of personal filing systems. International Journal of Man-Machine Studies, 36(1), pp. 97-126.

[3] Robertson, G., Czerwinski, M., Larson, K., Robbins, D. C., Thiel, D., \& Van Dantzich, M. (1998, November). Data mountain: using spatial memory for document management. In Proceedings of the 11th annual ACM symposium on User interface software and technology, pp. 153-162, ACM.

[4] Demian, P., \& Fruchter, R. (2005). Measuring relevance in support of design reuse from archives of building product models. Journal of computing in civil engineering, 19(2), pp. 119-136.

[5] Gray, C., \& Hughes, W. (2001). Building design management. Routledge.

[6] Leslie, H. G. (1996). Strategy for information in the AEC industry. INCIT 96 Proceedings: Bridging the Gap.

[7] Veeramani, D., \& Russell, J. (2000). Preparing the AEC industry for the knowledge economy. In Berkeley-Stanford Construction Engineering and Management Workshop: Defining a Research Agenda for AEC Process/Product Development in 2000 and Beyond.

[8] Ugwu, O. O. (2005). A service-oriented framework for sustainability appraisal and knowledge management. International Journal of ITConstruction ITCon (Electronic Journal), 10, pp. 245-263.

[9] Kiziltas, S., \& Akinci, B. (2009). Automated generation of customized field data collection templates to support information needs of cost estimators. Journal of Computing in Civil Engineering, 24(2), pp. 129-139.

[10] Brilakis, I. K., \& Soibelman, L. (2008). Shape-based retrieval of construction site photographs. Journal of Computing in Civil Engineering, 22(1), pp. 14-20.

[11] Caldas, C. H., Soibelman, L., \& Han, J. (2002). Automated classification of construction project documents. Journal of Computing in Civil Engineering, 16(4), pp. 234-243.

[12] Lin, K. Y., \& Soibelman, L. (2009). Incorporating domain knowledge and information retrieval techniques to develop an architectural/engineering/ construction online product search engine. Journal of Computing in Civil Engineering, 23(4), pp. 201-210.

[13] Rezgui, Y. (2006). Ontology-centered knowledge management using information retrieval techniques. Journal of Computing in Civil Engineering, 20(4), pp. 261-270.

[14] Eastman, C. M. (1999). Building product models: computer environments, supporting design and construction. CRC press.

[15] Emmitt, S., \& Ruikar, K. (2013). Collaborative design management. Routledge. 
[16] Austin, S., Baldwin, A., Li, B., \& Waskett, P. (2000). Analytical design planning technique (ADePT): a dependency structure matrix tool to schedule the building design process. Construction Management \& Economics, 18(2), pp. 173-182.

[17] Koo, B., \& Fischer, M. (2000). Feasibility study of 4D CAD in commercial construction. Journal of construction engineering and management, 126(4), pp. 251-260.

[18] Kunz, J. C., Christiansen, T. R., Cohen, G. P., Jin, Y., \& Levitt, R. E. (1998). The virtual design team. Communications of the ACM, 41(11), pp. 84-91.

[19] Pirolli, P., \& Card, S. (1999). Information foraging. Psychological review, 106(4), p. 643.

[20] Dominich, S. (2008). The modern algebra of information retrieval. Heidelberg: Springer.

[21] Card, S. K., Mackinlay, J. D., \& Shneiderman, B. (Eds.). (1999). Readings in information visualization: using vision to think. Morgan Kaufmann.

[22] Wu, I. C., \& Hsieh, S. H. (2012). A framework for facilitating multidimensional information integration, management and visualization in engineering projects. Automation in Construction, 23, pp. 71-86.

[23] Shaaban, S., Lockley, S., \& Elkadi, H. (2001). Information visualisation for the architectural practice. In Information Visualisation, 2001. Proceedings. Fifth International Conference on IEEE, pp. 43-50.

[24] Lansdale, M. W. (1988). The psychology of personal information management. Applied ergonomics, 19(1), pp. 55-66.

[25] Lansdale, M. W., Scrivener, S. A., \& Woodcock, A. (1996). Developing practice with theory in HCI: applying models of spatial cognition for the design of pictorial databases. International Journal of Human-Computer Studies, 44(6), pp. 777-799.

[26] Christmann, O., Carbonell, N., \& Richir, S. (2010). Visual search in dynamic $3 \mathrm{D}$ visualisations of unstructured picture collections. Interacting with Computers, 22(5), pp. 399-416.

[27] Rosson, M. B., \& Carroll, J. M. (2001). Usability engineering: scenariobased development of human-computer interaction. Elsevier.

[28] Brooke, J. (1996). SUS-A quick and dirty usability scale. Usability evaluation in industry, 189(194), pp. 4-7. 